\title{
The Smart Chain City
}

\author{
Walter Gaj Tripiano ${ }^{\mathrm{a}, 1}$ \\ a Polo Multimodal Pecem Investimentos, Fortaleza, Brazil
}

\begin{abstract}
The urban, social, industrial and technological evolution in recent years has forced designers, entrepreneurs and public entities to rethink the conceptual and operational logic for the construction of new urban settlements, according to the authentic principles of mutual respect between man and nature, eliminating all thought extremism and purely conceptual theories. In order to make their respective professional and life skills available to future generations, we have set up two international working groups, the ISCW and IS.Smart, in collaboration with the UFC universities of Fortaleza (Brazil), UIR of Rabat (Morocco) and UNITO of Turin (Italy), which for some years now, have been working to transform the notions of smart cities and smart buildings, combined with the new industrial revolution 4.0 and tokenomics, into concrete, multidisciplinary and educational activities. This publication, the result of the work developed in the last three years, intends to be an easy-to-read and application tool for all operators who wish to approach the "smart world" in a professional manner, especially in situations with serious housing shortage and still in the process of social and economic development. By reporting experiences and methodologies already field tested by the author in some working circumstances and that have given rise to case studies, similar to the "Polo Multimodal Pecem" (Brazil), the reader will find useful insights such as the definition of the smart concept and "smart" objectives, the approach to the project, the definition of evaluation parameters, operational examples of management, capital raising and crowdfunding, tokenomics and economy. I do believe that the future of the city will be increasingly linked to these issues, which have now become fundamental and necessary in the projection and planning processes of urban settlements.
\end{abstract}

Keywords. Smart cities, Block Chain

1 Head Innovation, Polo Multimodal Pecem Investimentos S.A., R. Vicente Linhares, 521 - Sala 2013 Aldeota - Fortaleza (CE. Brazil)www.polomultimodal.com; E-mail: studiogaj@hotmail.com 


\section{Introduction}

The case study of the Polo Multimodal Pecem, located in Brazil, in the State of Ceará, not far from Fortaleza (fig.1), can provide an interesting operational method, showing the steps that have defined the smart choices and procedures in planning the settlement.

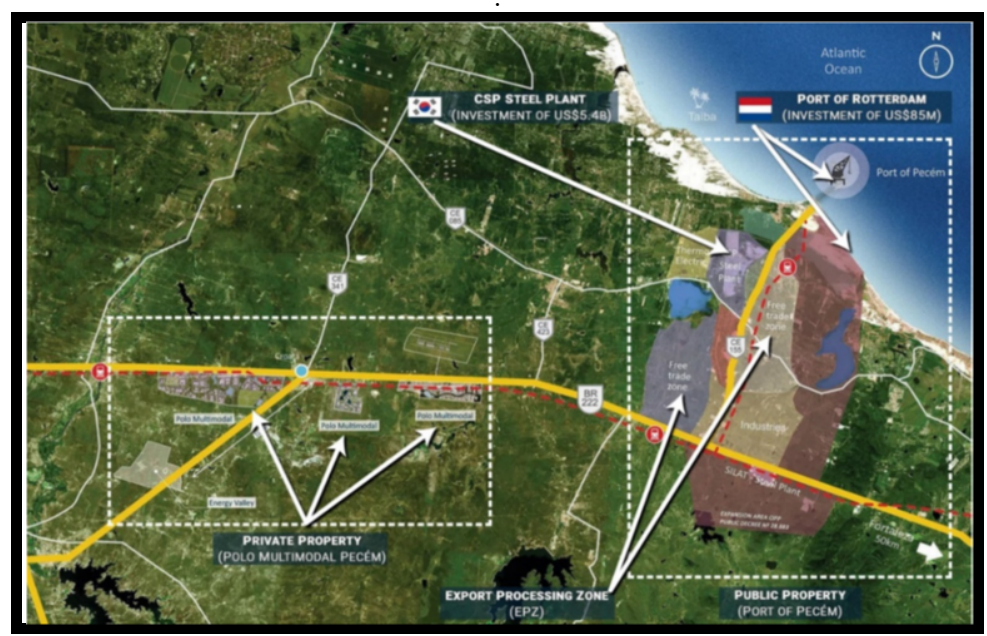

Figure 1. The Polo Multimodal Pecem location : Brazil, in the State of Ceará, not far from Fortaleza

The peculiarity of the settlement is that, in a single intelligent hub, all the different urban activities coexist: residential, health, logistics, production, commercial, development and research, cultural, sports and leisure, accommodation.

The project has been promoted by POLO MULTIMODAL PECEM INVESTIMENTS S.A., based in Fortaleza (Brazil), belonging to the Swiss company POLO SWISS DEVELOPMENT AG, and has been developed by a group of professionals in the area, including myself who, as Smart Concept Manager, was responsible for making both the design choices and the urban and architectural components "smart".

The Polo Multimodal Pecem emerged with the acquisition of the best lands (2000 hectares) located in a strategic point in the hinterland of Pecém (Fortaleza-Cearà), close to the EPZ (Export Processing Zone), a region in strong economic development also thanks to its proximity to the Panama Canal. The company "Port of Rotterdam" has recently acquired a stake in the company "Port of Pecém", to the demonstration that the choice made by the State of Ceará is of strategic importance. The "Smart Chain City" envisages the construction of industrial sheds, logistic, commercial and residential areas, as well as all infrastructure and related activities to meet the demand generated by the port and the large industries settled in the EPZ.The purpose of the project is to provide a competitive solution to the needs of small and medium-sized industries, not only locally, but also internationally. With this in mind, a strategic partnership was signed in Italy with Assoimprese (Association of Small and Medium-sized Industries), which aims to encourage the internationalization of the members in the areas of the Polo Multimodal and EPZ. 


\section{The project in detail}

To create a smart city from its foundations, it is indeed necessary to increase the logistic and production demand, fostering the installation of "smart" and sustainable companies, with the aim of providing all the tools to promote the development of the entire community.

As will be examined in detail below, the innovative process of the Polo Multimodal Pecem is made up of a digital platform called "Polo Digital Platform" which, based conceptually on block chain (smart contracts, smart assets, digital identity) and properly connected and distributed through technology "IoT" (Internet of Things), "Big Data" and "AI" (artificial intelligence), will integrate the control phases in the areas of infrastructure, services, technology and governance. Within this platform there is an exclusive section for companies, called "PoloSuit"; by accessing it companies will be able to take advantage, among other things, of specific services, that help in its simplified, transparent and effective installation, such as rent/purchase the property, company establishment, provision of public services, opening of current account, digital identification and any other necessary services.

The "smart" core of the project consists of an integrated management center called "Polo Chain Lab" (fig.2); an innovative center destined to house laboratories for applied technology in blockchain, advanced robotics, digital information and communication technology and 4.0 systems. This structure, intended for research and innovation, will be advantageous for companies, foundations and institutions, which will benefit from guidance, training, innovation and experimental development services.

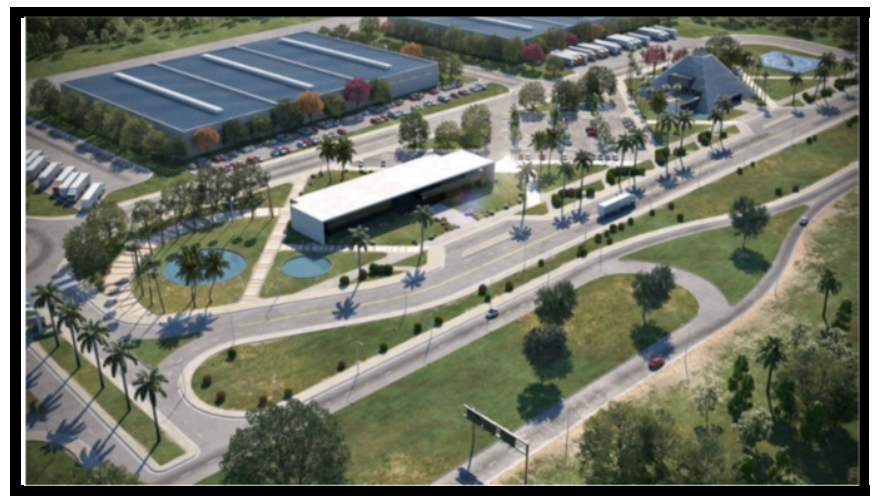

Figure 2. The Polo Chain Lab: The "smart" core of the project consists of an integrated management center, an innovative center destined to house laboratories for applied technology in blockchain, advanced robotics, digital information and communication technology and 4.0 systems.

Management through digital systems, with an integrated approach to data sharing, increases the decision-making capacity and its effectiveness. Each service will be more accurate and better tailored to individual needs, while at the same time being less costly to deliver: 
- the Polo will combine digital identity with smart assets using intelligent resources registered in the blockchain to verify and identify properties and related rights and obligations;

- IOT-powered AI devices integrated into a secure blockchain infrastructure will facilitate decision making and problem solving.

The project provides for the application of artificial intelligence algorithms to cameras located in the territory, so that an alert is automatically sent to the personnel of the local security operations center when abnormal situations or accidents are detected. The system is completed by specific "totems", also located in green areas, which allow emergency calls and communication with security personnel. In a smart city, where each person produces a large amount of data that must be shared on open platforms (open source), security means above all protecting the systems from "hacking" actions, either "white hat" or "black hat" (of good or bad intentions). When digital and physical systems converge as in smart cities, hacking represents a danger, and the more the city becomes hybrid, the more serious the danger is. The destructive potential for misappropriation of any smart system can be very frightening. Any urban dimension becomes a potential target for hackers: imagine, for example, uncontrolled self-driving cars, overly aggressive reactive architectures or a dangerously asynchronous distribution of energy. Hacking can, however, have an important positive function, both for safety and social reasons. By exploiting system gaps and invading them, white hats are able to uncover vulnerabilities and indicate new patterns, thus bringing innovation to the systems themselves. As paradoxical as it may seem, smart cities can stimulate creativity, if "hackable" to a predefined degree. For this reason, the study of a new computer model is in progress which, respecting the same principles used in the medical field in the analysis of the human body, pursues three objectives: redundancy, that is, a "reserve" of important parts of the system, capable of performing the task should the original part become incapacitated, such as pairs of limbs, eyes, ears in an animal being; segmentation, which allows the replacement of damaged elements before they can affect the healthy ones; proaction, which constantly subjects the system to hypothetical attacks by hackers and leads it to create, consequently, the necessary antivirus.

Encouraging the opening of platforms and data could stimulate new technological and cognitive paths, increase the commitment of residents and users, stimulate confrontations, lead to exchanges and collaborations, with the aim of increasing the level of urban culture. If we consider that open data and platforms have the same value for a smart city as accessible and public spaces have for a traditional city, the processes of urbanization are reduced to political issues that can only be managed through topdown and bottom-up processes. In short, the model applied to the Polo Multimodal Pecem is a new "mixed" type of security system, which provides a "classic" type of protection applied to a part of the data (especially sensitive data) and a protection created thanks to the top-down and bottom-up processes typical of the smart city.

The creation and management of the "PoloCoin" token will facilitate the exchange of goods and services within the smart city, ensuring greater security in business transactions between the companies settled and integrated in the supplychain.

The smart chain city will be powered entirely by clean energy produced by the Polo Energy Valley and will also include a waste recycling center; in addition, the project has been developed for: 
- $\quad$ increase the use of green industrial technologies and processes to achieve high levels of savings, both energy and resources used;

- achieve total management of the waste reduction system by promoting reuse and enhancement;

- carry out construction models with the purpose of obtaining specific certifications in the field of greenbuilding.

Since 2017, the project strategy has been in line with the 17 Sustainable Development Goals (SDOs) to be achieved by 2030, approved worldwide and adopted by the United Nations.

From which fundamental points was the project designed?

Once again we must look to the past to project ourselves into the future, starting from the ideas of a great American inventor, Buckminster Fuller, summarized as follows: "The function of what I call the science of design is to solve problems thanks to the contextual introduction of new artifacts whose availability will lead to their spontaneous use by human beings and therefore, simultaneously, will push man to abandon the behaviors and tools from which the problems originated " [1].

Developing a project in certain contexts or in some parts of the world necessarily leads to considering the process known as "leapfrogging", in which some situations of initial technological disadvantage can lead to considerable final benefits; for example, the lack of traditional data transmission networks, in the smart city area, can generate an advantage because fiber optic networks or new technologies can be installed immediately.

One might think at a first reading that the advent of the internet has cancelled the distances and physical places, giving people the possibility to work and operate in any place as it had been predicted by internet scholars since the nineties. Actually, urban space is expanding all over the world, the urban population is growing at a rate of 250,000 inhabitants per day, World Health Organization data indicate that by 2050 $75 \%$ of human beings will probably live in cities; this is because the essential element of human experience is the importance of physical interaction between people and the environment. As the Greek philosopher Aristotle wrote in the 4th century B.C. in "Politics", man is a social animal because he tends to aggregate with other individuals and to integrate himself into society, a concept taken up long after by the writer Italo Calvino in "The Invisible Cities": "The man who rides for a long time through wild lands is longing for a city" [2]. It is therefore essential that the design of physical spaces, architecture and urban planning, incorporate the digital network, combining virtual and real places, software and hardware. In other words, the smart city becomes the city where spaces are different from traditional ones because they are permeated by digital systems which transform the way people exchange experiences, socialize, communicate and live.

In the smart city, urban sensors transmit a huge amount of data that is processed by acting on the players responsible for modifying urban spaces: this creates a real test bench, a Big Data computing platform that links digital and physical spaces. The public physical space is thus transformed and renewed by users, who acquire a new sense of knowledge and power. Therefore, coherence in the planning of each part of the city is fundamental, because this will automatically achieve a very high standard of operation. "The word smart, or intelligent, refers to any digital technology used in a given urban context with the intention of producing new resources, improving existing ones, 
changing user behavior or ensuring other prospective improvements in terms of flexibility, security and sustainability" [3]. One of the most important features of a smart city is the immediate availability of all information, so that the urban system is not only controllable, but can also be optimized thanks to the advancement of information technology that allows connections, communications and interactions in real time. However, this flow of information, to provide advantages in terms of sustainability at the urban level (from the architecture equipped with people detector to public services developed to save resources), must have three essential elements: the instrumentation, basically a series of sensors that detect all the flows in the city; the analysis, data processing algorithms capable of understanding the present and predict future scenarios; the actuators, devices that can react in real time by making changes in physical space. This is how the "Internet of Things" (IoT) is born, that is, the property of each individual object connect bilaterally with other objects in order to transfer the structure of the Internet network to physical space. If we imagine, for example, applying a device to our home pantry, connected in turn with a sensor placed on the packaging of our favorite cookies, which is able to control the level of the product and, if they are finishing, order directly and exactly the same product to the nearest and most convenient grocery store, we would get a structure of interconnection of objects never existed before and similar to the Internet, capable of having important multiple effects.

This could lead to the creation and diffusion of a new generation of products, the socalled "every-ware", deeply linked to urban space; in other words, each urban and architectural element could be designed to work in a coherent and systematic way in order to achieve maximum efficiency in the use of resources.

However, in order to contain the risk that "too much intelligence of objects" will end up creating a certain anxiety in users, a new way of applying technologies is being introduced at the same time which, starting from the background, that is, the citizens, allows to improve its use thanks to the initiatives of each person. For this reason, new forms of aggregation are emerging among individuals with the aim of proposing different ideas and procedures, capable of transferring themselves from virtual space to the physical.

As a result, the dichotomy "bottom-up" / "top-down" arises, which will be discussed in detail later, a key aspect in the creation of a smart city. The bottom-up approach starts from a single element to create a system of increasing complexity, while the top-down approach starts from an overview that is then fragmented into detail.

For the execution of the project developed in Brazil, some strategies were applied:

\section{- Vision of the smart city}

Based on the relevant experiences and examples already existing in the world, both with regard to new smart cities and those that have become the result of smart processes, our vision is the meeting point between a technocentric and a holistic model. The path that leads to success is techno-sociological, and integrates the technological components through digital urban development projects shared on collaborative platforms by all involved. Although the purely technological component, services, digital applications and technological infrastructures, represent a significant part in the smart city model, about $60 \%$ of the total activities, we must consider that the flow of actions that feed urban growth is of non-technological, ecosystem and governmental nature, in order to have a smart, inclusive, sustainable and lasting city. 
- Type of smart city development

The holistic component presupposes a balance between a bottom-up type of development and a top-down model. The goal of the administrative body of a smart city should not be to centralize the decision-making process, but to make it open, inclusive, collaborative and strategically sound. Therefore, administrations participate both in projects related to the technological component and those belonging to the nontechnological component; for the latter it is necessary to provide the city with plans, procedures, standards, guidelines, programmatic documents, instructions and measures that make up a general strategic framework (vision, objectives, expected results, priority fields of action, etc.), and create specific management bodies to encourage the construction of a collaborative platform, which constantly involves inhabitants, companies, research centers and government agencies, through the organization of events (fairs, exhibitions, moments of discussion and confrontation, specific training courses, etc.) that make it possible to promote the smart city initiative, fostering awareness, sensitivity and training.

\section{- Smart city collaborative model}

Research, industry and government are the driving force behind the process of transformation of urban contexts in the smart city environment; to achieve a better performing model, civil society must also be included. This is what, in jargon, is called the "four drive collaborative model", where nodes, identified by groups of actors, connect to each other according to the type and amount of activities performed (open innovation and user-driven innovation).

- Smart city growth

The development of the smart city involves a close collaboration between organizations of different types and civil society, embedded in an inclusive, heterogeneous and highly participatory ecosystem. The presence of a collective intelligence stimulates the activation of new collaborations and partnerships, enriching the knowledge needed to foster the transformation of projects and making available to the system, sharing them, the creativity and skills of individuals (smart community). From the encounter between individual and collective contribution emerges the intelligence of society, which allows the transformations in an intelligent key and the progressive integration of new digital services and technological infrastructures within the territory.

\section{- Smart city concept}

It is necessary to reason in a multidimensional way. On the one hand, the process of intelligent transformation is fed mainly by digital urban development projects that promote energy efficiency and a better use of natural resources, acting mainly in buildings, energy networks and transportation systems; on the other hand, it is necessary to work within a broader and more transversal field of action, which allows the extension of the use of ICT to other sectors where the problems to be addressed are not only related to energy and environmental sustainability, but also to the financial, business, commercial, health, cultural and sports aspects. 
What are the operational elements that a team of designers should consider, and from which they should start, when creating a smart city? Or, more specifically, what are the main thematic areas on which architects and engineers should focus their work to propose new models compatible with the smart city concept?

- Mobility

For the Polo Multimodal Pecem project a smart mobility was conceived, carried out both through structural investments and through low cost initiatives that act in social innovation and in the awareness of users/inhabitants.

Structural investments include:

Differentiated road network design according to the use destinations of the areas and the type of transport used;

Versatility of the road network for future transformations, as the smart city is dynamic due to technological innovations and the evolution programs of its inhabitants;

Creation of "park and ride", areas designed to improve the quality of life of the entire urban area, increasing the use of more sustainable means of transport and decreasing noise levels in storage/production areas.

\section{- $\quad$ Energy efficiency}

In the Polo Multimodal Pecem project the use of passive energy saving systems has been planned, aimed at ensuring comfort levels without the need for artificially produced energy, thanks only to the characteristics of the building's coating (shape, architecture, exposure, orientation, thermal insulation and thermal mass, protection from solar radiation, materials) and the way heat is transported inside the rooms or outside the building (air, sun, sky). The passive conception, better defined in the building environment with the name of bioclimatic architecture, produces design solutions in which architecture and natural context are closely related to each other, starting from a detailed analysis of the site to determine the climatic agents capable of bringing advantages or against which it is necessary to protect oneself.

\section{- Environmental sustainability}

Use of "green energy" and reduction of $\mathrm{CO} 2$ emissions. The Polo Multimodal Pecem project developed green solutions mainly in two directions, the first, of a general nature, considering the construction "on the ground" of a photovoltaic park with large production capacity at the service of the hub itself, the second, of a punctual type, going to define " smart " systems of energy management of buildings and built environments and implementing photovoltaic / wind power plants with lower production capacity connected to the main network of energy distribution in "in/out" mode.

- $\quad$ Smart living

In the smart city, smart living allows urban centers to find, through specific innovative solutions, an important "market launch"; for example, it is possible to advertise an international call to collect ideas that help to identify specific territorial marketing, or to reshape the city's image and design its logo. Web and 2.0 communication are central 
when it comes to smart living; the Polo Multimodal Pecem project has developed some specific applications designed to improve and enhance the use and appreciation of local resources, as well as increase the opportunities offered to spend free time ("social networking").

\section{- Smart People}

Participation, involvement, dialogue, interaction, listening: there can be no smart city if the foundations for good coexistence, open dialogue between citizens and administrators are missing. In the Polo Multimodal Pecem project, through the specific platform called "PoloDigital" (fig.3), and in order to remain in constant contact with the administration and develop common projects, an operational manual is available to users that illustrates specific tools such as the general survey, particular surveys, focus group, surveys about the place where the service is provided, online surveys, collection of demonstrations, suggestions and complaints, petitions and signature campaign. Through PoloDigital, a crowdfunding platform will be created that will improve the collaboration between the administration, the citizen and the companies, aiming at identifying resources and financing the agreed social projects.

- Smart Economy

The Polo Multimodal Pecem project envisages the creation of a specific center called "PoloChain Lab" that deals with investments in the "Knowledge Economy", focusing on research and innovation, encouraging processes of internationalization, sharing knowledge and enhancing creative talent. Investing in the Knowledge Economy means managing the transformation process of the smart city, promoting a synergistic system in which private companies, public agencies, research institutes, universities, science parks, collaborate to raise the technological level, creating a stimulating environment for everyone.

\section{- $\quad$ Smart Governance}

The Polo Multimodal Pecem offers, through the "Integrated Management Center" interconnected with the platforms "PoloChain Lab" and "PoloSuit", a "governance", a transparent and friendly promoter to share through open data the flows of information that you will receive daily from users, with the aim of simplifying the administration, digitizing processes and procedures to reach e-government. A smart city must have a smart administration, capable of defining its own strategy, be in harmony with the active citizenship, to aspire to a better and long-term future. In fact, it is through smart governance that smart service projects are developed, from those in the educationalcultural, business, commercial and even mobility, leisure and sports fields [4]. 


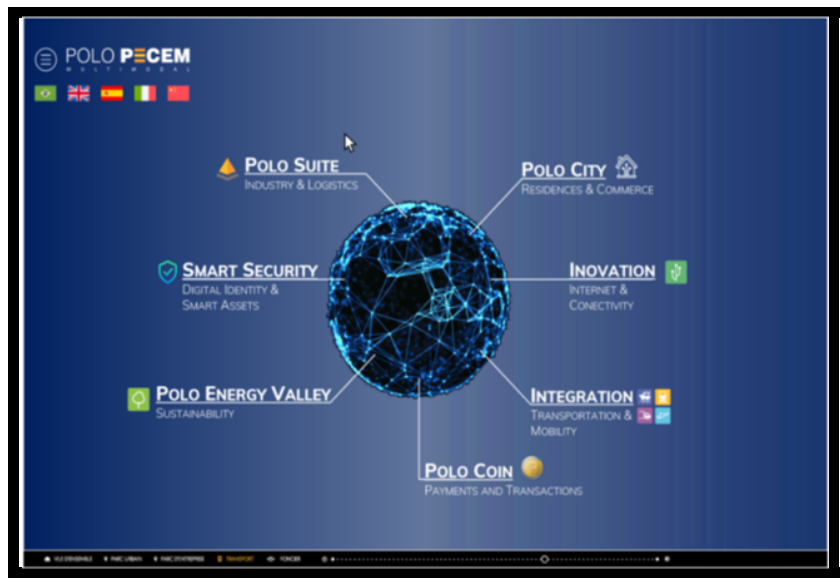

Figure 3. The Polo Digital platform : Participation, involvement, dialogue, interaction, listening: there can be no smart city if the foundations for good coexistence, open dialogue between citizens and administrators are missing. In the Polo Multimodal Pecem project, through the specific platform.

\section{References}

[1] Richard Buckminster Fuller and Kiyoshi Kuromiya, Cosmography. A Posthumous Scenario for the Future of Humanity, Macmillan, New York 1992, p.8.

[2] Italo Calvino, Le città invisibili, Giulio Einaudi editore s.p.a., Turin 1972, p.16.

[3] Francesca Bria and Evgeny Morozov, Ripensare la smart city, Codice Edizioni,Turin 2018, p.9.

[4] Walter Gaj Tripiano. Smart City Designing the smart city The case study of the Polo Multimodal Pecem Brazil. Fuoril[u]ogo. 2020 Sep 